\title{
Ester and amide derivatives of rhodamine B exert cytotoxic effects on different human tumor cell lines
}

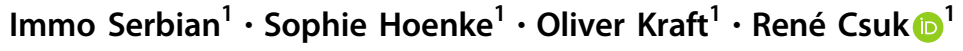

Received: 15 April 2020 / Accepted: 11 June 2020 / Published online: 22 June 2020

(c) The Author(s) 2020

\begin{abstract}
Three esters of rhodamine B (1-3) differing in their alkyl chain lengths as well as several rhodamine B amides (4-9) were synthesized in good yields and tested for their cytotoxicity in SRB assays employing several human tumor cell lines. The rhodamine $\mathrm{B}$ esters were unselective but showed cytotoxicity of as low as $\mathrm{EC}_{50}=0.15 \pm 0.02 \mu \mathrm{M}$. The rhodamine $\mathrm{B}$ amides were slightly less cytotoxic but showed good selectivity against MCF-7 and A2780 tumor cell lines. Especially a morpholinyl derivative $\mathbf{4}$ was $\sim 20$ time more cytotoxic for MCF-7 than for nonmalignant NIH 3T3 cells.
\end{abstract}

\section{Graphical Abstract}

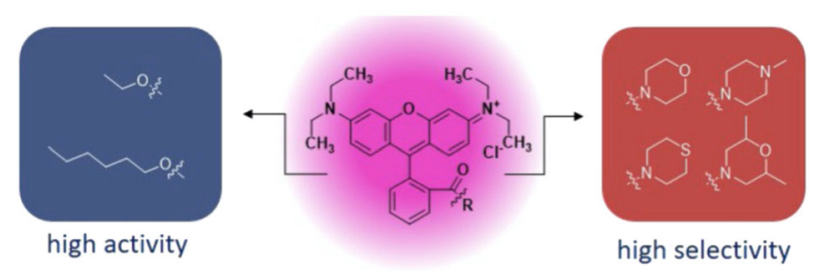

Keywords Rhodamine B $\cdot$ Rhodamine B amide $\cdot$ Rhodamine B esters $\cdot$ Cytotoxicity $\cdot$ SRB assay

\section{Introduction}

Rhodamines are widely used in fluorescence microscopy to stain cell compartments especially mitochondria (Guo et al. 2018; Johnson et al. 1980; Talib et al. 2019; Wang et al. 2018; Zhang et al. 2020). The preferential transport of these xanthylium scaffold based dyes into mitochondria has previously been used to selectively direct cytotoxic compounds into mitochondria (Kahnt et al. 2018; Sommerwerk et al.

Supplementary information The online version of this article (https:// doi.org/10.1007/s00044-020-02591-8) contains supplementary material, which is available to authorized users.

René Csuk

rene.csuk@chemie.uni-halle.de

1 Martin-Luther-University Halle-Wittenberg, Organic Chemistry, Kurt-Mothes-Str. 2, D-06120 Halle (Saale), Germany
2017; Wiemann et al. 2018; Wolfram et al. 2018a, 2018b; Xie et al. 2013). While rhodamine B displays only minor cytotoxicity, di- or triterpenoid conjugates holding an attached rhodamine B moiety with or without a spacer between these parts of the conjugate proved highly cytotoxic. Several of these "mitocanic" conjugates held an even nanomolar activity for human tumor cell lines (Sommerwerk et al. 2017; Wolfram et al. 2018a, 2018b). It has also been shown that these mitocans led to a controlled cell death; some of them could distinguish very well between malignant and nonmalignant cells thus providing a high selectivity for malignant human tumor cell lines (Kahnt et al. 2018; Sommerwerk et al. 2017; Wiemann et al. 2018; Wolfram et al. 2018a, 2018b). As a consequence, mitochondria have emerged as a major drug target inasmuch as they can induce a programmed cell death in human tumor cells (Costantini et al. 2000; Fulda 2010; Galluzzi et al. 2006; Gogvadze et al. 2009a, 2009b; Neuzil et al. 2013). While the exact mechanism still remains unclear it appears 
that the presence of a lipophilic cation in the conjugates is one of the necessary prerequisites for achieving good cytotoxicity (Neuzil et al. 2013; Sommerwerk et al. 2017). In the case of triterpenoic acids, there are indications that both the choice of the respective triterpenoic scaffold (maslinic acid is better than, e.g., oleanolic acid), the spacer (piperazinyl spacered compounds holding a rhodamine B dye are more cytotoxic than analogs holding an ethylenediamine spacer) and the type of cation (rhodamine B derived compounds are more cytotoxic than, for example, analogs holding a malachite green derived moiety) are of decisive importance (Kahnt et al. 2018; Sommerwerk et al. 2017; Wiemann et al. 2018; Wolfram et al. 2018a, 2018b). Furthermore, it must also be pointed out that the presence of a rhodamine B residue in a molecule does not guarantee the achievement of high cytotoxicity in a conjugate (Wiemann et al. 2018).

Triterpenoic acids are notoriously poorly soluble in water which limits their bioavailability (Csuk and Deigner 2019; Shakurova et al. 2020; Song et al. 2019). The formation of rhodamine B conjugates significantly increases their solubility, but these molecules are nevertheless not perfect according to Lipinski's "rule of five" rule (Oprea 2002; Walters et al. 1999). This gave rise to the question to what extent the triterpenoid part in these molecules could be avoided at all. Since no "simple" derivatives of rhodamine B have been evaluated for their cytotoxicity against human tumor cell lines we decided to prepare several esters and amides of rhodamine $\mathrm{B}$ and to investigate their cytotoxic effects (Fig. 1 and Scheme 1).

\section{Results and discussion}

The synthesis of the rhodamine B drug conjugates was straightforward: for the synthesis of the esters (Mai and Allison 1983; Mottram et al. 2012; Rashid and Horobin 1990; Tansil et al. 2011; Wieker et al. 1987; Yu et al. 2001) 1-3, to a solution of rhodamine B acyl chloride either ethanol, hexanol or eicosanol were added in the presence of triethylamine to afford the esters in yields ranging from 48 to $85 \%$. For the synthesis of the amides (Beija et al. 2011; Bui et al. 2014; Dauner et al. 2016; Del Secco et al. 2017; May et al. 2012; Preston et al. 2018; Sodano et al. 2018) 4-9 rhodamine B acyl chloride was allowed to react with an excess of the corresponding amine; thereby the products were obtained in isolated yields ranging from 68 to $92 \%$.

The compounds were subjected to sulforhodamine B assays (SRB) to evaluate their cytotoxicity; the results of these assays are summarized in Table 1 .

As a result, compounds 1-9 were cytotoxic for all human tumor cell lines; their $\mathrm{EC}_{50}$ values ranged from excellent $0.15 \pm 0.02 \mu \mathrm{M}$ for compound 2 to very low $\mathrm{EC}_{50}$ values of

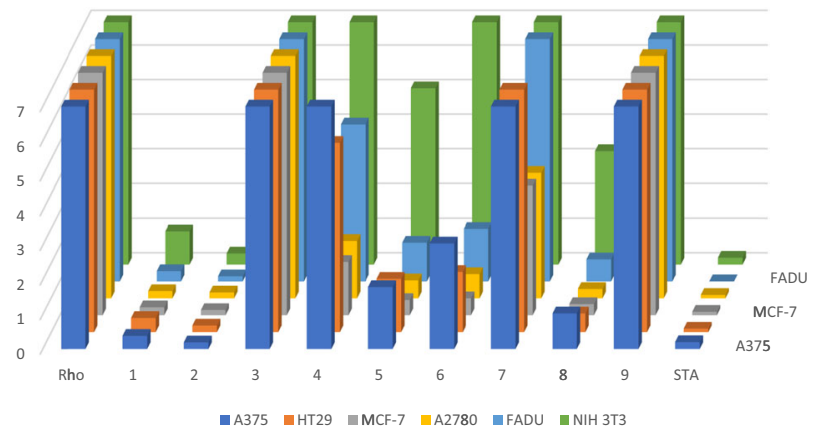

Fig. 1 Cytotoxicity of compounds 1-9, STA and Rho; 3D bar chart representation (cutoff at $7 \mu \mathrm{M}$ )

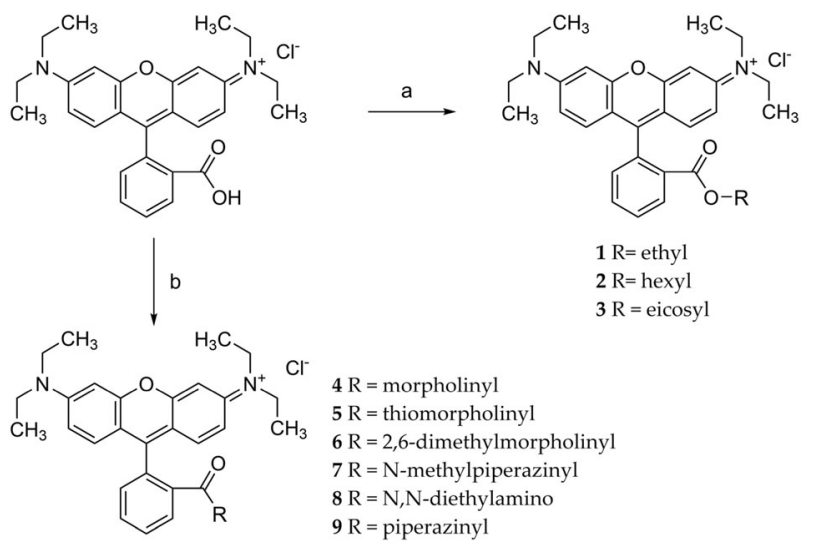

Scheme 1 Synthesis of several rhodamine B drug conjugates: a 1. $(\mathrm{COCl})_{2}, \mathrm{DMF}, \mathrm{CH}_{2} \mathrm{Cl}_{2}$ 2. ethanol/1-hexanol/1-eicosanol, $\mathrm{NEt}_{3}$ (2 eq.) $0{ }^{\circ} \mathrm{C}$ b 1 . $(\mathrm{COCl})_{2}, \mathrm{DMF}, \mathrm{CH}_{2} \mathrm{Cl}_{2}$ 2. morpholine/thiomorpholine/2,6dimethylmorpholine/N-methylpiperazine/N, N-diethylamine/piperazine, $0{ }^{\circ} \mathrm{C}, 30 \mathrm{~min}$

$>20 \mu \mathrm{M}$ for the eicosyl ester 3. This somewhat surprising result suggests that transport through the membrane(s) is not the limiting factor, since it is known that rhodamine B esters with hydrophobic moieties permeate lipid membranes faster than their hydrophilic analogs (Melikyan et al. 1996; Rokitskaya et al. 2008, 2018).

While the rhodamine B esters are highly active holding $\mathrm{EC}_{50}$ values lower than $1 \mu \mathrm{M}$ these compounds lack selectivity (Table 2). Although it would make sense to study a homologous series of these esters holding different chain lengths with the aim of finding a "magic" chain length where cytotoxicity is highest, we have refrained from doing so. We justify this by the fact that the selectivity factors of the esters (as compared to those of the amides) are too low, and a sufficiently large differentiation between malignant and nonmalignant cells is not likely.

However, the rhodamine B amides also showed high cytotoxic effects with $\mathrm{EC}_{50}$ values between $0.27 \pm 0.01$ and $17.34 \pm 0.8 \mu \mathrm{M}$. Especially compound $\mathbf{8}$ was very cytotoxic holding $\mathrm{EC}_{50}$ values lower than $1 \mu \mathrm{M}$ while the other 
Table 1 Cytotoxicity of compounds 1-9 and rhodamine $\mathrm{B}$ (Rho) $\left(\mathrm{EC}_{50}\right.$ values in $\mu \mathrm{M}$ from SRB assays after $72 \mathrm{~h}$ of treatment, the values are averaged from three independent experiments performed each in triplicate, confidence interval CI $=95 \%$; mean \pm standard mean error, cutoff $30 \mu \mathrm{M}$ )

\begin{tabular}{lllllll}
\hline Compound & A375 & HT29 & MCF-7 & A2780 & FADU & NIH 3T3 \\
\hline Rho & $>30$ & $>30$ & $>30$ & $>30$ & $>30$ & $>30$ \\
$\mathbf{1}$ & $0.38 \pm 0.02$ & $0.41 \pm 0.04$ & $0.23 \pm 0.03$ & $0.21 \pm 0.01$ & $0.30 \pm 0.02$ & $0.96 \pm 0.05$ \\
$\mathbf{2}$ & $0.19 \pm 0.01$ & $0.19 \pm 0.03$ & $0.15 \pm 0.02$ & $0.17 \pm 0.01$ & $0.15 \pm 0.01$ & $0.32 \pm 0.02$ \\
$\mathbf{3}$ & $>20$ & $>20$ & $>20$ & $>20$ & $>20$ & $>20$ \\
$\mathbf{4}$ & $7.09 \pm 0.3$ & $5.46 \pm 0.2$ & $1.54 \pm 0.3$ & $1.66 \pm 0.1$ & $4.53 \pm 0.2$ & $>30$ \\
$\mathbf{5}$ & $1.79 \pm 0.1$ & $1.54 \pm 0.1$ & $0.44 \pm 0.1$ & $0.52 \pm 0.03$ & $1.12 \pm 0.1$ & $5.09 \pm 0.2$ \\
$\mathbf{6}$ & $3.05 \pm 0.20$ & $1.74 \pm 0.04$ & $0.49 \pm 0.08$ & $0.70 \pm 0.14$ & $1.52 \pm 0.10$ & $7.92 \pm 0.30$ \\
$\mathbf{7}$ & $16.05 \pm 0.6$ & $17.34 \pm 0.8$ & $3.74 \pm 0.3$ & $3.62 \pm 0.2$ & $11.78 \pm 0.5$ & $>30$ \\
$\mathbf{8}$ & $1.03 \pm 0.03$ & $0.54 \pm 0.02$ & $0.32 \pm 0.04$ & $0.27 \pm 0.01$ & $0.64 \pm 0.03$ & $3.27 \pm 0.08$ \\
$\mathbf{9}$ & $>30$ & $>30$ & $17.8 \pm 3.9$ & $26.4 \pm 2.1$ & $>30$ & $>30$ \\
STA & $0.2 \pm 0.02$ & $0.1 \pm 0.01$ & $0.1 \pm 0.01$ & $0.1 \pm 0.05$ & $0.008 \pm 0.001$ & $0.2 \pm 0.02$ \\
\hline
\end{tabular}

Human cancer cell lines: A375 (epithelial melanoma), HT29 (colorectal adenocarcinoma), MCF-7 (breast adenocarcinoma), A2780 (ovarian carcinoma), FaDu (squamous cell carcinoma); nonmalignant: NIH 3T3 (mouse fibroblasts). Staurosporine (STA) was used as a positive standard
Table 2 Selectivity factors of compounds $\mathbf{1 - 9}$ with $\mathrm{S}_{\text {tumor cell line }}=\mathrm{EC}_{50}$ (NIH 3T3) / $\mathrm{EC}_{50}$ (tumor cell line)

\begin{tabular}{lrrrrr}
\hline Compound & A375 & HT29 & MCF-7 & A2780 & FaDu \\
\hline $\mathbf{1}$ & 2.5 & 2.3 & 4.2 & 4.6 & 3.2 \\
$\mathbf{2}$ & 1.7 & 1.7 & 2.1 & 1.9 & 2.1 \\
$\mathbf{4}$ & $>4.2$ & $>5.5$ & $>19.5$ & $>18.1$ & $>6.6$ \\
$\mathbf{5}$ & 2.8 & 3.3 & 11.6 & 9.8 & 4.5 \\
$\mathbf{6}$ & 2.6 & 4.6 & 16.2 & 11.3 & 5.2 \\
$\mathbf{7}$ & $>1.9$ & $>1.7$ & $>8.0$ & $>8.3$ & $>2.5$ \\
$\mathbf{8}$ & 3.2 & 6.1 & 10.2 & 12.1 & 5.1 \\
$\mathbf{9}$ & - & - & $>1.7$ & $>1.1$ & - \\
\hline
\end{tabular}

rhodamine B amides were slightly less cytotoxic. Interestingly enough, piperazine derived 9 was significantly less active than N-methyl-piperazine derived compound 7. As far as the selectivity factors of all compounds are concerned, it is of interest to note that the "simple" rhodamine $\mathrm{B}$ amides showed a significant selectivity for human tumor cell lines MCF-7 (breast adenocarcinoma) and A2780 (ovarian carcinoma). Especially the morpholinyl derivative 4 exhibited with $S=19.5$ the highest selectivity factor for MCF-7 and with $S=18.1$ for A2780 tumor cells.

\section{Conclusion}

Rhodamine derived dyes are widely used to stain the mitochondria of cells; although rhodamine B is classified as potentially carcinogenic it does not show cytotoxicity up to $30 \mu \mathrm{M}$. To get a deeper insight into the cytotoxicity of rhodamine $\mathrm{B}$ derived conjugates, three different esters of rhodamine B (1-3) differing in their alkyl chain length as well as six amides (4-9) were prepared in good yields and tested for their cytotoxicity using several human tumor cell lines. Esters and amides of rhodamine B with triterpenoids have previously been shown to be highly cytotoxic for tumor cells holding $\mathrm{EC}_{50}$ values in the $\mathrm{nM}$ region (Sommerwerk et al. 2017; Wiemann et al. 2018; Wolfram et al. 2018a). Hence it would be of interest to evaluate whether a triterpenoid scaffold is necessary for cytotoxicity or if simple esters and amides of rhodamine B may also perform as well as in SRB assays. As a result, the ethyl and hexyl ester of rhodamine B showed against MCF-7 tumor cells $\mathrm{EC}_{50}$ values as low as 0.23 and $0.15 \mu \mathrm{M}$, respectively. An eicosyl derivative, however, whose lipophilicity is even more close to that of triterpenoids did not show even moderate cytotoxicity although the long nonpolar alkyl chain might be able to interfere with membranes. Furthermore, the rhodamine B esters also lack selectivity.

Surprisingly, the morpholinyl derived rhodamine B amide was not as cytotoxic as the rhodamine B esters (albeit being in a low $\mu \mathrm{M}$ range with $\mathrm{EC}_{50}$ values ranging from 0.44 to $3.76 \mu \mathrm{M}$ for MCF-7 breast adenocarcinoma cells) but showed good selectivity factors against tumor cells of 8-19.5 for MCF-7 tumor cells. The calculated selectivity factors, however, might even be higher but due to the cutoff limit of the assay the exact values could not be determined.

Depending on their substitution pattern, triterpenoid rhodamine conjugates are quite cytotoxic and several of them are highly selective against tumor cells. The good selectivity of the "simple" morpholinyl derived compounds as in contrast to the complex triterpenoids cannot be explained. One might assume, they are able to interfere with NF-kB, caspase-3/8/9 and or mTOR/PI3K/Akt-pathways which also are known to be altered by triterpenoids.

Ongoing investigations will provide evidence whether these compounds are able to trigger permeabilization of the mitochondrial membrane due to a change in the mitochondrial membrane potential or to interfere with the mitochondrial permeability transition pore. 


\section{Experimental}

The equipment as well as the details of the cytotoxic evaluation can be found in the supplementary materials file.

\section{Synthesis}

\section{Rhodamine B chloride (= N-(9-(2-(chlorocarbonyl)phenyl)- 6-(diethylamino)-3H-xanthen-3-ylidene)- $\mathrm{N}$ - ethylethanaminium chloride)}

The fluorescent dye rhodamine B $(10.0 \mathrm{~g}, 22.3 \mathrm{mmol})$ was dissolved in dry $\mathrm{CH}_{2} \mathrm{Cl}_{2}(250 \mathrm{~mL})$, treated with oxalyl chloride $(8.84 \mathrm{~mL})$ at $0{ }^{\circ} \mathrm{C}$. One drop of dry DMF was added, and the solution was allowed to warm up to room temperature. After completion of the reaction, the solvent was removed under reduced pressure. The residue was dissolved in dry $\mathrm{CH}_{2} \mathrm{Cl}_{2}(50 \mathrm{~mL})$, and the solution was concentrated again to remove excess oxalyl chloride. Yielding rhodamine B chloride $(11.0 \mathrm{~g}, 99 \%)$ as a purple solid which is used without further purification

\section{General procedure for the synthesis of the rhodamine B esters 1-3}

Rhodamine $\mathrm{B}$ acyl chloride $(500 \mathrm{mg}, 1.0 \mathrm{mmol})$ was dissolved in dry $\mathrm{CH}_{2} \mathrm{Cl}_{2}(50 \mathrm{~mL})$, and at $0{ }^{\circ} \mathrm{C}$ the corresponding alcohol (1 eq.) and triethylamine $(2 \mathrm{mmol}, 0.28 \mathrm{~mL})$ were added. The reaction progress was monitored by TLC, and after complete conversion the solvent was removed under reduced pressure. The crude reaction mixture was purified by column chromatography $\left(\mathrm{SiO}_{2}, \mathrm{CHCl}_{3} / \mathrm{MeOH}\right)$ to yield the rhodamine B esters (1-3) each as a dark purple solid.

\section{General procedure for the synthesis of the rhodamine B amides 4-9}

Rhodamine B acyl chloride $(500 \mathrm{mg}, 1.0 \mathrm{mmol}$ ) was dissolved in dry $\mathrm{CH}_{2} \mathrm{Cl}_{2}(50 \mathrm{~mL})$. The corresponding amine (4 eq.) was added slowly at $0{ }^{\circ} \mathrm{C}$. The mixture was stirred for $30 \mathrm{~min}$, the solvent was removed under reduced pressure, and the residue was purified by column chromatography $\left(\mathrm{SiO}_{2}, \mathrm{CHCl}_{3} / \mathrm{MeOH}\right)$ to yield the rhodamine $\mathrm{B}$ amides (4-9) each as a dark purple solid.

\section{3,6-Bis(diethylamino)-9[2-(ethyloxy)carbonyl]-xanthylium chloride (1)}

Yield: $433 \mathrm{mg}(85 \%)$; m.p. $124-127{ }^{\circ} \mathrm{C} ; R_{F}=0.39\left(\mathrm{SiO}_{2}\right.$, $\mathrm{CHCl}_{3} / \mathrm{MeOH}, 8: 2$ ); IR (ATR): $\nu=3322 \mathrm{w}, 3166 \mathrm{~m}, 2981 \mathrm{~m}$, $1712 \mathrm{~s}, 1644 \mathrm{~m}, 1589 \mathrm{~s}, 1543 \mathrm{~s}, 1502 \mathrm{~m}, 1463 \mathrm{~s}, 1409 \mathrm{~s}$, $1390 \mathrm{~m}, 1366 \mathrm{~m}, 1334 \mathrm{~s}, 1262 \mathrm{~s}, 1240 \mathrm{~s}, 1178 \mathrm{~s}, 1131 \mathrm{~s}, 1077 \mathrm{~s}$, $1043 \mathrm{~m}, 1010 \mathrm{~s}, 919 \mathrm{~m}, 828 \mathrm{~m}, 759 \mathrm{~m}, 708 \mathrm{~m}, 665 \mathrm{~m}, 576 \mathrm{~m} \mathrm{~cm}^{-1}$;
${ }^{1} \mathrm{H}$ NMR $\left(400 \mathrm{MHz}, \mathrm{CDCl}_{3}\right): \delta=8.21(\mathrm{dd}, J=7.9,1.4 \mathrm{~Hz}$, $1 \mathrm{H}, 5 \mathrm{H}), 7.73(\mathrm{dt}, J=7.5,1.4 \mathrm{~Hz}, 1 \mathrm{H}, 7 \mathrm{H}), 7.66(\mathrm{dt}, J=$ $7.7,1.4 \mathrm{~Hz}, 1 \mathrm{H}, 6 \mathrm{H}), 7.22(\mathrm{dd}, J=7.5,1.4 \mathrm{~Hz}, 1 \mathrm{H}, 8 \mathrm{H})$, $7.00\left(\mathrm{~d}, J=9.5 \mathrm{~Hz}, 2 \mathrm{H}, 12 \mathrm{H}+12^{\prime} \mathrm{H}\right), 6.84(\mathrm{dd}, J=9.5$, $\left.2.4 \mathrm{~Hz}, 2 \mathrm{H}, 13 \mathrm{H}+13^{\prime} \mathrm{H}\right), 6.72(\mathrm{~d}, J=2.4 \mathrm{~Hz}, 2 \mathrm{H}, 15 \mathrm{H}+$ $\left.15^{\prime} \mathrm{H}\right), 3.99\left(\mathrm{q}, J=7.1 \mathrm{~Hz}, 2 \mathrm{H}, 2^{\prime} \mathrm{H}\right), 3.58(\mathrm{q}, J=7.2 \mathrm{~Hz}$, $\left.8 \mathrm{H}, \quad 17 \mathrm{H}+17^{\prime} \mathrm{H}+17^{\prime \prime} \mathrm{H}+17^{\prime \prime \prime} \mathrm{H}\right), \quad 1.25 \quad(\mathrm{t}, \quad J=7.1 \mathrm{~Hz}$, $\left.12 \mathrm{H}, 18 \mathrm{H}+18^{\prime} \mathrm{H}+18^{\prime \prime} \mathrm{H}+18^{\prime \prime \prime} \mathrm{H}\right), 0.99(\mathrm{t}, J=7.1 \mathrm{~Hz}$, $3 \mathrm{H}, 1 \mathrm{H}) \mathrm{ppm} ;{ }^{13} \mathrm{C}$ NMR $\left(101 \mathrm{MHz}, \mathrm{CDCl}_{3}\right): \delta=165.0(\mathrm{C}-$ 3), 158.9 (C-4), 157.7 (C-16 + C-16'), 155.5 (C-14 + C14'), 133.4 (C-9), 132.9 (C-7), 131.3 (C-12 + C-12'), 131.2 (C-5), 130.3 (C-6), 130.1 (C-8), 130.1 (C-10), 114.2 (C$\left.13+\mathrm{C}-13^{\prime}\right), 113.5\left(\mathrm{C}-11+\mathrm{C}-11^{\prime}\right), 96.2\left(\mathrm{C}-15+\mathrm{C}-15^{\prime}\right)$, $61.5(\mathrm{C}-2), 46.1\left(\mathrm{C}-17+\mathrm{C}-17^{\prime}+\mathrm{C}-17^{\prime \prime}+\mathrm{C}-17^{\prime \prime \prime}\right), 13.7$ (C-1), $12.6\left(\mathrm{C}-18+\mathrm{C}-18^{\prime}+\mathrm{C}-18^{\prime \prime}+\mathrm{C}-18^{\prime \prime \prime}\right)$ ppm; MS (ESI, MeOH): $\mathrm{m} / z=471.4\left(100 \%,[\mathrm{M}]^{+}\right)$; analysis calcd for $\mathrm{C}_{30} \mathrm{H}_{35} \mathrm{~N}_{2} \mathrm{O}_{3} \mathrm{Cl}$ (507.07): C 71.06, $\mathrm{H}$ 6.96, N 5.52; found: $\mathrm{C}$ 70.76, H 7.18, N 5.31.

\section{3,6-Bis(diethylamino)-9[2-(hexyloxy)carbonyl]-xanthylium chloride (2)}

Yield: $397 \mathrm{mg}$ (70\%); m.p. $159-162{ }^{\circ} \mathrm{C} ; R_{F}=0.41\left(\mathrm{SiO}_{2}\right.$, $\mathrm{CHCl}_{3} / \mathrm{MeOH}, 8: 2$ ); IR (ATR): $\nu=3063 \mathrm{w}, 2956 \mathrm{w}, 2929 \mathrm{~m}$, $2858 \mathrm{w}, 1716 \mathrm{~m}, 1646 \mathrm{~m}, 1584 \mathrm{~s}, 1529 \mathrm{~m}, 1507 \mathrm{~m}, 1481 \mathrm{~m}$, $1466 \mathrm{~s}, 1435 \mathrm{~m}, 1411 \mathrm{~s}, 1395 \mathrm{~m}, 1334 \mathrm{~s}, 1272 \mathrm{~s}, 1245 \mathrm{~s}$, $1197 \mathrm{~m}, 1177 \mathrm{~s}, 1160 \mathrm{~s}, 1130 \mathrm{~s}, 1072 \mathrm{~s}, 1008 \mathrm{~m}, 977 \mathrm{~m}, 922 \mathrm{~m}$, $824 \mathrm{~m}, 758 \mathrm{~m}, 707 \mathrm{~m}, 681 \mathrm{~s}, 667 \mathrm{~m}, 579 \mathrm{~m} \mathrm{~cm}^{-1} ;{ }^{1} \mathrm{H} \mathrm{NMR}$ $\left(400 \mathrm{MHz}, \mathrm{CDCl}_{3}\right): \delta=8.28(\mathrm{dd}, J=7.8,1.3 \mathrm{~Hz}, 1 \mathrm{H}, 9 \mathrm{H})$, $7.80(\mathrm{dt}, J=7.5,1.4 \mathrm{~Hz}, 1 \mathrm{H}, 11 \mathrm{H}), 7.73(\mathrm{dt}, J=7.7$, $1.4 \mathrm{~Hz}, 1 \mathrm{H}, 10 \mathrm{H}), 7.30(\mathrm{dd}, J=7.5,1.3 \mathrm{~Hz}, 1 \mathrm{H}, 12 \mathrm{H}), 7.07$ $\left(\mathrm{d}, J=9.4 \mathrm{~Hz}, 2 \mathrm{H}, 16 \mathrm{H}+16^{\prime} \mathrm{H}\right), 6.90(\mathrm{dd}, J=9.4,2.2 \mathrm{~Hz}$, $\left.2 \mathrm{H}, 17 \mathrm{H}+17^{\prime} \mathrm{H}\right), 6.82\left(\mathrm{~d}, J=2.3 \mathrm{~Hz}, 2 \mathrm{H}, 19 \mathrm{H}+19^{\prime} \mathrm{H}\right)$, $3.99(\mathrm{t}, J=6.6 \mathrm{~Hz}, 2 \mathrm{H}, 6 \mathrm{H}), 3.64(\mathrm{q}, J=7.2 \mathrm{~Hz}, 8 \mathrm{H}, 21 \mathrm{H}$ $\left.+21^{\prime} \mathrm{H}+21^{\prime \prime} \mathrm{H}+21^{\prime \prime \prime} \mathrm{H}\right), 1.43-1.35(\mathrm{~m}, 2 \mathrm{H}, 5 \mathrm{H}), 1.32(\mathrm{t}$, $\left.J=7.1 \mathrm{~Hz}, 12 \mathrm{H}, 22 \mathrm{H}+22^{\prime} \mathrm{H}+22^{\prime \prime} \mathrm{H}+22^{\prime \prime \prime} \mathrm{H}\right), 1.28-1.07$ $(\mathrm{m}, 6 \mathrm{H}, 4 \mathrm{H}+3 \mathrm{H}+2 \mathrm{H}), 0.82(\mathrm{t}, J=6.9 \mathrm{~Hz}, 3 \mathrm{H}, 1 \mathrm{H}) \mathrm{ppm}$; ${ }^{13} \mathrm{C} \mathrm{NMR}\left(101 \mathrm{MHz}, \mathrm{CDCl}_{3}\right): \delta=165.2(\mathrm{C}-7), 158.9(\mathrm{C}-8)$, 157.8 (C-20 + C-20'), $155.6\left(\mathrm{C}-18+\mathrm{C}-18^{\prime}\right), 133.4(\mathrm{C}-13)$, 133.0 (C-11), 131.3 (C-16 + C-16'), 131.3 (C-9), 130.4 (C10), 130.3 (C-12), 130.2 (C-14), $114.3\left(\mathrm{C}-17+\mathrm{C}-17^{\prime}\right)$, $113.6\left(\mathrm{C}-15+\mathrm{C}-15^{\prime}\right), 96.4\left(\mathrm{C}-19+\mathrm{C}-19^{\prime}\right), 65.8(\mathrm{C}-6), 46.2$ $\left(\mathrm{C}-21+\mathrm{C}-21^{\prime}+\mathrm{C}-21^{\prime \prime}+\mathrm{C}-21^{\prime \prime \prime}\right), 31.3$ (C-3), 28.3 (C-5), 25.5 (C-4), 22.4 (C-2), 14.0 (C-1), 12.7 (C-22 + C-22' + C$\left.22^{\prime \prime}+\mathrm{C}-22^{\prime \prime \prime}\right)$ ppm; MS (ESI, MeOH): $m / z=527.5(100 \%$, $[\mathrm{M}]^{+}$); analysis calcd for $\mathrm{C}_{34} \mathrm{H}_{43} \mathrm{ClN}_{2} \mathrm{O}_{3}$ (563.18): C 72.51, H 7.70, N 4.97; found: C 72.38, H 7.96, N 4.73.

3,6-Bis(diethylamino)-9[2-(eicosyloxy)carbonyl]-xanthylium chloride (3)

Yield: $362 \mathrm{mg}(48 \%) ;$ m.p. $144-146{ }^{\circ} \mathrm{C} ; R_{F}=0.38\left(\mathrm{SiO}_{2}\right.$, $\mathrm{CHCl}_{3} / \mathrm{MeOH}, \quad 9: 1$ ); IR (ATR): $\nu=2919 \mathrm{~m}, 2850 \mathrm{~m}$, 
$1721 \mathrm{~m}, 1645 \mathrm{~m}, 1586 \mathrm{~s}, 1553 \mathrm{~m}, 1528 \mathrm{~m}, 1489 \mathrm{~m}, 1467 \mathrm{~s}$, $1433 \mathrm{~m}, 1412 \mathrm{~s}, 1396 \mathrm{~m}, 1381 \mathrm{~m}, 1333 \mathrm{~s}, 1272 \mathrm{~s}, 1249 \mathrm{~s}$, $1198 \mathrm{~m}, 1179 \mathrm{~s}, 1160 \mathrm{~m}, 1132 \mathrm{~s}, 1075 \mathrm{~s}, 1040 \mathrm{~m}, 1011 \mathrm{~m}$, $974 \mathrm{~m}, 923 \mathrm{~m}, 824 \mathrm{~m}, 708 \mathrm{~m}, 682 \mathrm{~s}, 668 \mathrm{~m} \mathrm{~cm}^{-1}$; ${ }^{1} \mathrm{H} \mathrm{NMR}$ $\left(500 \mathrm{MHz} \mathrm{CDCl}_{3}\right): \delta=8.29-8.25(\mathrm{~m}, 1 \mathrm{H}, 23 \mathrm{H}), 7.79(\mathrm{dt}$, $J=7.6,1.4 \mathrm{~Hz}, 1 \mathrm{H}, 25 \mathrm{H}), 7.72(\mathrm{dt}, J=7.7,1.3 \mathrm{~Hz}, 1 \mathrm{H}$, 24H4), $7.30(\mathrm{dd}, J=7.6,1.3 \mathrm{~Hz}, 1 \mathrm{H}, 26 \mathrm{H}), 7.06$ (d, $\left.J=9.3 \mathrm{~Hz}, 2 \mathrm{H}, 30 \mathrm{H}+30^{\prime} \mathrm{H}\right), 6.86(\mathrm{~d}, J=2.5 \mathrm{~Hz}, 2 \mathrm{H}$, $\left.21 \mathrm{H}+21^{\prime} \mathrm{H}\right), 6.85-6.83\left(\mathrm{~m}, 2 \mathrm{H}, 33 \mathrm{H}+33^{\prime} \mathrm{H}\right), 3.98(\mathrm{t}$, $J=6.7 \mathrm{~Hz}, 2 \mathrm{H}, 20 \mathrm{H}), 3.62\left(\mathrm{q}, J=7.4 \mathrm{~Hz}, 8 \mathrm{H}, 35 \mathrm{H}+35^{\prime}\right.$ $\left.\mathrm{H}+35^{\prime \prime} \mathrm{H}+35^{\prime \prime \prime} \mathrm{H}\right), \quad 1.42-1.37\left(\mathrm{~m}, 2 \mathrm{H}, 18 \mathrm{H}_{\mathrm{a}}+18 \mathrm{H}_{\mathrm{b}}\right)$, $1.31\left(\mathrm{t}, J=7.1 \mathrm{~Hz}, 12 \mathrm{H}, 36 \mathrm{H}+36^{\prime} \mathrm{H}+36^{\prime \prime} \mathrm{H}+36^{\prime \prime \prime} \mathrm{H}\right)$, $1.29-1.26\left(\mathrm{~m}, 2 \mathrm{H}, 2 \mathrm{H}_{\mathrm{a}}+2 \mathrm{H}_{\mathrm{b}}\right), 1.24-1.14\left(\mathrm{~m}, 28 \mathrm{H}, 3 \mathrm{H}_{\mathrm{a}}+\right.$ $\left.3 \mathrm{H}_{\mathrm{b}}+4 \mathrm{H}-17 \mathrm{H}\right), 1.13-1.07\left(\mathrm{~m}, 2 \mathrm{H}, 19 \mathrm{H}_{\mathrm{a}}+19 \mathrm{H}_{\mathrm{b}}\right), 0.86(\mathrm{t}$, $J=6.9 \mathrm{~Hz}, 3 \mathrm{H}, 1 \mathrm{H}) \mathrm{ppm} ;{ }^{13} \mathrm{C} \mathrm{NMR}\left(126 \mathrm{MHz}, \mathrm{CDCl}_{3}\right): \delta$ $=165.2(\mathrm{C}-21), 158.9(\mathrm{C}-22), 157.8 \quad\left(\mathrm{C}-34+\mathrm{C}-34^{\prime}\right)$, $155.6\left(\mathrm{C}-32+\mathrm{C}-32^{\prime}\right), 133.4$ (C-27), 133.0 (C-25), 131.3 $\left(\mathrm{C}-30+\mathrm{C}-30^{\prime}\right), 131.2(\mathrm{C}-23), 130.3(\mathrm{C}-24), 130.3(\mathrm{C}-26)$, $130.2(\mathrm{C}-28), 114.1\left(\mathrm{C}-21+\mathrm{C}-21^{\prime}\right), 113.6\left(\mathrm{C}-29+\mathrm{C}-29^{\prime}\right)$, $96.5\left(\mathrm{C}-33+\mathrm{C}-33^{\prime}\right), 65.8(\mathrm{C}-20), 46.1\left(\mathrm{C}-35+\mathrm{C}-35^{\prime}+\right.$ $\left.\mathrm{C}-35^{\prime \prime}+\mathrm{C}-35^{\prime \prime \prime}\right), 31.9$ (C-3), 29.7-29.2 (C-4-C-17), 28.3 (C-18), 25.8 (C-19), 22.7 (C-2), 14.1 (C-1), 12.6 (C-36 $\left.+\mathrm{C}-36^{\prime}+\mathrm{C}-36^{\prime \prime}+\mathrm{C}-36^{\prime \prime \prime}\right)$ ppm; MS (ESI, MeOH): $\mathrm{m} / z=$ $723.7\left(100 \%,[\mathrm{M}]^{+}\right)$; analysis calcd for $\mathrm{C}_{48} \mathrm{H}_{71} \mathrm{ClN}_{2} \mathrm{O}_{3}$ (759.56): C 75.90, H 9.42, N 3.69; found: C 75.75, H 9.61, N 3.55 .

\section{3,6-Bis(diethylamino)-9[2-(1-morpholinyl)carbonyl]- xanthylium chloride (4)}

Yield: $503 \mathrm{mg}(92 \%)$; m.p. $>250{ }^{\circ} \mathrm{C} ; R_{F}=0.34\left(\mathrm{SiO}_{2}\right.$, $\mathrm{CHCl}_{3} / \mathrm{MeOH}, 9: 1$ ); IR (ATR): $\nu=3356 \mathrm{w}, 3060 \mathrm{w}, 2976 \mathrm{w}$, $2933 \mathrm{w}, 2871 \mathrm{w}, 2795 \mathrm{w}, 2605 \mathrm{w}, 2498 \mathrm{w}, 1644 \mathrm{~m}, 1624 \mathrm{~m}$, $1584 \mathrm{~s}, 1528 \mathrm{~m}, 1508 \mathrm{~m}, 1481 \mathrm{~s}, 1465 \mathrm{~s}, 1446 \mathrm{~s}, 1431 \mathrm{~s}, 1411 \mathrm{~s}$, $1394 \mathrm{~s}, 1334 \mathrm{~s}, 1271 \mathrm{~s}, 1245 \mathrm{~s}, 1196 \mathrm{~m}, 1178 \mathrm{~s}, 1161 \mathrm{~s}, 1132 \mathrm{~s}$, $1094 \mathrm{~m}, 1071 \mathrm{~s}, 1008 \mathrm{~m}, 976 \mathrm{~m}, 921 \mathrm{~m}, 822 \mathrm{~m}, 740 \mathrm{~s}, 682 \mathrm{~s}$, $655 \mathrm{~m}, 657 \mathrm{~m} \mathrm{~cm}^{-1} ;{ }^{1} \mathrm{H}$ NMR $\left(500 \mathrm{MHz}, \mathrm{CDCl}_{3}\right): \delta=$ 7.65-7.62 (m, 2H, 7H+8H), 7.50-7.47 (m, 1H, 5H), $7.32-7.29(\mathrm{~m}, 1 \mathrm{H}, 6 \mathrm{H}), 7.19\left(\mathrm{~d}, J=9.5 \mathrm{~Hz}, 2 \mathrm{H}, 12 \mathrm{H}+12^{\prime}\right.$ $\mathrm{H}), 6.95\left(\mathrm{dd}, J=9.6,2.4 \mathrm{~Hz}, 2 \mathrm{H}, 13 \mathrm{H}+13^{\prime} \mathrm{H}\right), 6.73(\mathrm{~d}, J$ $\left.=2.5 \mathrm{~Hz}, 2 \mathrm{H}, 15 \mathrm{H}+15^{\prime} \mathrm{H}\right), 3.60(\mathrm{q}, J=7.2 \mathrm{~Hz}, 8 \mathrm{H}, 17 \mathrm{H}$ $\left.+17^{\prime} \mathrm{H}+17^{\prime \prime} \mathrm{H}+17^{\prime \prime \prime} \mathrm{H}\right), 3.45-3.40$ (m, 4H, $\left.1 \mathrm{H}+1^{\prime} \mathrm{H}\right)$, 3.39-3.29 (m, 4H, $\left.2 \mathrm{H}+2^{\prime} \mathrm{H}\right), 1.28(\mathrm{t}, J=7.1 \mathrm{~Hz}, 12 \mathrm{H}$, $\left.18 \mathrm{H}+18^{\prime} \mathrm{H}+18^{\prime \prime} \mathrm{H}+18^{\prime \prime \prime} \mathrm{H}\right) \mathrm{ppm} ;{ }^{13} \mathrm{C} \mathrm{NMR}(126 \mathrm{MHz}$, $\left.\mathrm{CDCl}_{3}\right): \delta=167.4$ (C-3), 157.7 (C-14), 155.7 (C-4), 155.6 $\left(\mathrm{C}-16+\mathrm{C}-16^{\prime}\right), 135.0(\mathrm{C}-9), 132.0\left(\mathrm{C}-12+\mathrm{C}-12^{\prime}\right), 130.7$ (C-10), 130.3 (C-7), 130.2 (C-6), 130.1 (C-8), 127.6 (C-5), $114.2\left(\mathrm{C}-13+\mathrm{C}-13^{\prime}\right), 113.7\left(\mathrm{C}-11+\mathrm{C}-11^{\prime}\right), 96.3(\mathrm{C}-15+$ C-15'), 66.6 (C-1), 48.0 (C-2a), $46.2\left(\mathrm{C}-17+\mathrm{C}-17^{\prime}+\mathrm{C}-17^{\prime}\right.$ $\left.'+\mathrm{C}-17^{\prime \prime \prime}\right), 42.2(\mathrm{C}-2 \mathrm{~b}), 12.6\left(\mathrm{C}-18+\mathrm{C}-18^{\prime}+\mathrm{C}-18^{\prime \prime}+\mathrm{C}-\right.$ $18^{\prime \prime \prime}$ ) ppm; MS (ESI, MeOH): $m / z=512.3\left(100 \%,[\mathrm{M}]^{+}\right)$; analysis calcd for $\mathrm{C}_{32} \mathrm{H}_{38} \mathrm{ClN}_{3} \mathrm{O}_{3}$ (548.12): C 70.12, H 6.99, N 7.67; found: C 69.84, H 7.17, N 7.49.
3,6-Bis(diethylamino)-9[2-(1-thiomorpholinyl)carbonyl]xanthylium chloride (5)

Yield: $471 \mathrm{mg}(83 \%)$; m.p. $233{ }^{\circ} \mathrm{C} ; R_{F}=0.35\left(\mathrm{SiO}_{2}, \mathrm{CHCl}_{3} /\right.$ MeOH, 9:1); IR (ATR): $\nu=3311 \mathrm{w}, 3060 \mathrm{w}, 2972 \mathrm{w}, 2906 \mathrm{w}$, $1628 \mathrm{~m}, 1585 \mathrm{~s}, 1527 \mathrm{~m}, 1508 \mathrm{~m}, 1481 \mathrm{~m}, 1467 \mathrm{~s}, 1411 \mathrm{~s}$, $1394 \mathrm{~m}, 1335 \mathrm{~s}, 1305 \mathrm{~m}, 1273 \mathrm{~s}, 1245 \mathrm{~s}, 1178 \mathrm{~s}, 1131 \mathrm{~s}, 1093 \mathrm{~m}$, $1072 \mathrm{~s}, 1049 \mathrm{~m}, 1036 \mathrm{~m}, 1008 \mathrm{~m}, 951 \mathrm{~m}, 920 \mathrm{~m}, 742 \mathrm{~m}, 682 \mathrm{~s}$, $665 \mathrm{~m} \mathrm{~cm}^{-1} ;{ }^{1} \mathrm{H}$ NMR $\left(500 \mathrm{MHz}, \mathrm{CDCl}_{3}\right): \delta=7.64-7.60$ $(\mathrm{m}, 2 \mathrm{H}, 7 \mathrm{H} 7+8 \mathrm{H}), 7.48-7.45(\mathrm{~m}, 1 \mathrm{H}, 5 \mathrm{H}), 7.29-7.27(\mathrm{~m}$, $1 \mathrm{H}, 6 \mathrm{H}), 7.17\left(\mathrm{~d}, J=9.5 \mathrm{~Hz}, 2 \mathrm{H}, 12 \mathrm{H}+12^{\prime} \mathrm{H}\right), 6.93(\mathrm{dd}$, $\left.J=9.5,2.4 \mathrm{~Hz}, 2 \mathrm{H}, 13 \mathrm{H}+13^{\prime} \mathrm{H}\right), 6.72(\mathrm{~d}, J=2.5 \mathrm{~Hz}, 2 \mathrm{H}$, $\left.15 \mathrm{H}+15^{\prime} \mathrm{H}\right), 3.59\left(\mathrm{qd}, J=7.3,2.1 \mathrm{~Hz}, 8 \mathrm{H}, 17 \mathrm{H}+17^{\prime} \mathrm{H}+\right.$ $\left.17^{\prime \prime} \mathrm{H}+17^{\prime \prime \prime} \mathrm{H}\right), 3.58-3.46\left(\mathrm{~m}, 4 \mathrm{H}, 2 \mathrm{H}+2^{\prime} \mathrm{H}\right), 2.47-2.37$ $\left(\mathrm{m}, 4 \mathrm{H}, 1 \mathrm{H}+1^{\prime} \mathrm{H}\right), 1.27\left(\mathrm{t}, J=7.1 \mathrm{~Hz}, 12 \mathrm{H}, 18 \mathrm{H}+18^{\prime} \mathrm{H}+\right.$ $\left.18^{\prime \prime} \mathrm{H}+18^{\prime \prime \prime} \mathrm{H}\right) \mathrm{ppm} ;{ }^{13} \mathrm{C} \mathrm{NMR}\left(126 \mathrm{MHz}, \mathrm{CDCl}_{3}\right): \delta=$ 167.7 (C-3), 157.7 (C-14), $155.6\left(\mathrm{C}-16+\mathrm{C}-16^{\prime}\right), 155.6(\mathrm{C}-$ 4), 135.3 (C-9), 132.1 (C-12+C-12'), 130.5 (C-10), 130.3 (C-7), 130.2 (C-6), 130.0 (C-8), 127.3 (C-5), 114.2 (C-13+ C-13'), $113.7\left(\mathrm{C}-11+\mathrm{C}-11^{\prime}\right), 96.3\left(\mathrm{C}-15+\mathrm{C}-15^{\prime}\right), 50.0(\mathrm{C}-$ $2 \mathrm{a}), 46.2\left(\mathrm{C}-17+\mathrm{C}-17^{\prime}+\mathrm{C}-17^{\prime \prime}+\mathrm{C}-17^{\prime \prime \prime}\right), 44.1$ (C-2b), $27.6(\mathrm{C}-1), 12.6\left(\mathrm{C}-18+\mathrm{C}-18^{\prime}+\mathrm{C}-18^{\prime \prime}+\mathrm{C}-18^{\prime \prime \prime}\right) \mathrm{ppm} ; \mathrm{MS}$ (ESI, $\mathrm{MeOH}): m / z=528.4\left(100 \%,[\mathrm{M}]^{+}\right)$; analysis calcd for $\mathrm{C}_{32} \mathrm{H}_{38} \mathrm{ClN}_{3} \mathrm{O}_{2} \mathrm{~S}$ (564.19): C 68.13, H 6.79, N 7.45, S 5.68; found: C 67.90, H 6.82, N 7.29, S 5.56.

\section{3,6-Bis(diethylamino)-9[2-(1-(2,6-dimethylmorpholinyl) carbonyl)]-xanthylium chloride (6)}

Yield: $483 \mathrm{mg}$ (84\%); m.p. $179-181^{\circ} \mathrm{C} ; R_{F}=0.38\left(\mathrm{SiO}_{2}\right.$, $\mathrm{CHCl}_{3} / \mathrm{MeOH}, 9: 1$ ); IR (ATR): $\nu=2974 \mathrm{w}, 2933 \mathrm{w}, 2871 \mathrm{w}$, $1644 \mathrm{~m}, 1629 \mathrm{~m}, 1585 \mathrm{~s}, 1528 \mathrm{~m}, 1507 \mathrm{~m}, 1481 \mathrm{~m}, 1467 \mathrm{~m}$, $1455 \mathrm{~m}, 1431 \mathrm{~m}, 1411 \mathrm{~s}, 1394 \mathrm{~m}, 1331 \mathrm{~s}, 1272 \mathrm{~s}, 1245 \mathrm{~s}$, $1196 \mathrm{~m}, 1178 \mathrm{~s}, 1159 \mathrm{~s}, 1131 \mathrm{~s}, 1094 \mathrm{~m}, 1071 \mathrm{~s}, 1039 \mathrm{~m}$, $1009 \mathrm{~m}, 975 \mathrm{~m}, 920 \mathrm{~m}, 823 \mathrm{~m}, 682 \mathrm{~s}, 666 \mathrm{~m} \mathrm{~cm}^{-1} ;{ }^{1} \mathrm{H} \mathrm{NMR}$ $\left(500 \mathrm{MHz}, \mathrm{CDCl}_{3}\right): \delta=7.69-7.65(\mathrm{~m}, 2 \mathrm{H}, \quad 7 \mathrm{H}+8 \mathrm{H})$, 7.51-7.48 (m, 1H, 5H), 7.36-7.33 (m, 1H, 6H), 7.26-7.19 $\left(\mathrm{m}, 2 \mathrm{H}, 12 \mathrm{H}+12^{\prime} \mathrm{H}\right), 6.96-6.87\left(\mathrm{~m}, 2 \mathrm{H}, 13 \mathrm{H}+13^{\prime} \mathrm{H}\right)$, 6.83-6.79 (m, $\left.2 \mathrm{H}, 15 \mathrm{H}+15^{\prime} \mathrm{H}\right), 4.12(\mathrm{~d}, J=13.2 \mathrm{~Hz}, 1 \mathrm{H}$, $\left.2 \mathrm{H}_{\mathrm{a}}\right), 3.66-3.57\left(\mathrm{~m}, 8 \mathrm{H}, 17 \mathrm{H}+17^{\prime} \mathrm{H}+17^{\prime \prime} \mathrm{H}+17^{\prime \prime \prime} \mathrm{H}\right)$, $3.42\left(\mathrm{~d}, J=12.7 \mathrm{~Hz}, 1 \mathrm{H}, 2^{\prime} \mathrm{H}_{\mathrm{a}}\right), 3.22-3.12(\mathrm{~m}, 2 \mathrm{H}, 1 \mathrm{H})$, $3.09-3.00\left(\mathrm{~m}, 2 \mathrm{H}, 1^{\prime} \mathrm{H}\right), 2.58\left(\mathrm{t}, J=11.9 \mathrm{~Hz}, 1 \mathrm{H}, 2 \mathrm{H}_{\mathrm{b}}^{\prime}\right)$, $2.21\left(\mathrm{t}, J=11.6 \mathrm{~Hz}, 1 \mathrm{H}, 2 \mathrm{H}_{\mathrm{b}}\right), 1.30(\mathrm{t}, J=7.1 \mathrm{~Hz}, 12 \mathrm{H}$, $\left.18 \mathrm{H}+18^{\prime} \mathrm{H}+18^{\prime \prime} \mathrm{H}+18^{\prime \prime \prime} \mathrm{H}\right), 1.08-0.98(\mathrm{~m}, 6 \mathrm{H}, 19 \mathrm{H}+$ $\left.19^{\prime} \mathrm{H}\right) \mathrm{ppm} ;{ }^{13} \mathrm{C}$ NMR $\left(126 \mathrm{MHz}, \mathrm{CDCl}_{3}\right): \delta=167.3(\mathrm{C}-3)$, 157.7 (C-14), 155.8 (C-16), $155.6\left(\mathrm{C}-16^{\prime}\right), 155.5$ (C-4), 135.2 (C-9), 132.4 (C-12), 131.9 (C-12'), 130.3 (C-10), 130.3 (C-7), 130.3 (C-6), 130.0 (C-8), 127.6 (C-5), 114.1 (C-13), $113.9\left(\mathrm{C}-13^{\prime}\right), 113.7$ (C-11), $113.6\left(\mathrm{C}-11^{\prime}\right), 96.5(\mathrm{C}-$ $\left.15+\mathrm{C}-15^{\prime}\right), 71.9(\mathrm{C}-1), 71.7\left(\mathrm{C}-1^{\prime}\right), 52.9(\mathrm{C}-2), 47.1\left(\mathrm{C}-2^{\prime}\right)$, $46.1\left(\mathrm{C}-17+\mathrm{C}-17^{\prime}+\mathrm{C}-17^{\prime \prime}+\mathrm{C}-17^{\prime \prime \prime}\right), 18.6(\mathrm{C}-19), 18.4$ $\left(\mathrm{C}-19^{\prime}\right), 12.60\left(\mathrm{C}-18+\mathrm{C}-18^{\prime}+\mathrm{C}-18^{\prime \prime}+\mathrm{C}-18^{\prime \prime \prime}\right) \mathrm{ppm} ; \mathrm{MS}$ (ESI, $\mathrm{MeOH}): m / z=540.4\left(100 \%,[\mathrm{M}]^{+}\right)$; analysis calcd for 
$\mathrm{C}_{34} \mathrm{H}_{42} \mathrm{ClN}_{3} \mathrm{O}_{3}$ (576.18): C 70.88, H 7.35, N 7.29; found: C $70.59, \mathrm{H} 7.53, \mathrm{~N} 7.01$.

\section{3,6-Bis(diethylamino)-9[2-(1-(4-methylpiperazinyl) carbonyl)]-xanthylium chloride (7)}

Yield: $384 \mathrm{mg}$ (68\%); m.p. $>250{ }^{\circ} \mathrm{C} ; \mathrm{R}_{F}=0.29\left(\mathrm{SiO}_{2}\right.$, $\mathrm{CHCl}_{3} / \mathrm{MeOH}, 9: 1$ ); IR (ATR): $\nu=3061 \mathrm{w}, 2975 \mathrm{w}, 2899 \mathrm{w}$, $2869 \mathrm{w}, 2717 \mathrm{w}, 2468 \mathrm{w}, 1629 \mathrm{~m}, 1583 \mathrm{~s}, 1528 \mathrm{~m}, 1508 \mathrm{~m}$, $1481 \mathrm{~s}, 1466 \mathrm{~s}, 1411 \mathrm{~s}, 1394 \mathrm{~s}, 1334 \mathrm{~s}, 1299 \mathrm{~m}, 1272 \mathrm{~s}, 1245 \mathrm{~s}$, $1196 \mathrm{~m}, 1177 \mathrm{~s}, 1159 \mathrm{~s}, 1131 \mathrm{~s}, 1113 \mathrm{~s}, 1093 \mathrm{~m}, 1071 \mathrm{~s}, 1009 \mathrm{~s}$, 976m, 921m, 822m, 739s, 681s, $656 \mathrm{~m} \mathrm{~cm}^{-1}$; ${ }^{1} \mathrm{H}$ NMR $\left(500 \mathrm{MHz}, \mathrm{CDCl}_{3}\right): \delta=7.74-7.70(\mathrm{~m}, 1 \mathrm{H}, 5 \mathrm{H}), 7.64(\mathrm{dt}$, $J=7.6,1.4 \mathrm{~Hz}, 1 \mathrm{H}, 7 \mathrm{H}), 7.60(\mathrm{dt}, J=7.5,1.4 \mathrm{~Hz}, 1 \mathrm{H}, 8 \mathrm{H})$, $7.26(\mathrm{dd}, J=7.5,1.4 \mathrm{~Hz}, 1 \mathrm{H}, 6 \mathrm{H}), 7.14(\mathrm{~d}, J=9.5 \mathrm{~Hz}, 2 \mathrm{H}$, $\left.12 \mathrm{H}+12^{\prime} \mathrm{H}\right), 6.89\left(\mathrm{dd}, J=9.6,2.4 \mathrm{~Hz}, 2 \mathrm{H}, 13 \mathrm{H}+13^{\prime} \mathrm{H}\right)$, $6.69\left(\mathrm{~d}, J=2.4 \mathrm{~Hz}, 2 \mathrm{H}, 15 \mathrm{H}+15^{\prime} \mathrm{H}\right), 3.65-3.50(\mathrm{~m}, 8 \mathrm{H}$, $\left.17 \mathrm{H}+17^{\prime} \mathrm{H}+17^{\prime \prime} \mathrm{H}+17^{\prime \prime \prime} \mathrm{H}\right), 3.51-3.43\left(\mathrm{~m}, 4 \mathrm{H}, 2 \mathrm{H}+2^{\prime}\right.$ $\mathrm{H}), 2.71-2.54\left(\mathrm{~m}, 4 \mathrm{H}, 1 \mathrm{H}+1^{\prime} \mathrm{H}\right), 2.52-2.43(\mathrm{~m}, 3 \mathrm{H}, 19 \mathrm{H})$, $1.26\left(\mathrm{t}, \quad J=7.2 \mathrm{~Hz}, 12 \mathrm{H}, 18 \mathrm{H}+18^{\prime} \mathrm{H}+18^{\prime \prime} \mathrm{H}+18^{\prime \prime \prime} \mathrm{H}\right)$ ppm; ${ }^{13} \mathrm{C}$ NMR $\left(126 \mathrm{MHz}, \mathrm{CDCl}_{3}\right): \delta=167.3(\mathrm{C}-3), 157.7$ (C-14), 156.0 (C-4), 155.6 (C-16+C-16'), 134.9 (C-9), $131.9\left(\mathrm{C}-12+\mathrm{C}-12^{\prime}\right), 130.6(\mathrm{C}-10), 130.3(\mathrm{C}-7), 130.1(\mathrm{C}-$ 6), 129.9 (C-8), 128.1 (C-5), $114.1\left(\mathrm{C}-13+\mathrm{C}-13^{\prime}\right), 113.7$ $\left(\mathrm{C}-11+\mathrm{C}-11^{\prime}\right), 96.2\left(\mathrm{C}-15+\mathrm{C}-15^{\prime}\right), 53.4\left(\mathrm{C}-1+\mathrm{C}-1^{\prime}\right)$, $46.1\left(\mathrm{C}-17+\mathrm{C}-17^{\prime}+\mathrm{C}-17^{\prime \prime}+\mathrm{C}-17^{\prime \prime \prime}\right), 44.8$ (C-19), 40.5 $\left(\mathrm{C}-2+\mathrm{C}-2^{\prime}\right), 12.6\left(\mathrm{C}-18+\mathrm{C}-18^{\prime}+\mathrm{C}-18^{\prime \prime}+\mathrm{C}-18^{\prime \prime \prime}\right) \mathrm{ppm}$; MS (ESI, $\mathrm{MeOH}): m / z=525.5\left(100 \%,[\mathrm{M}]^{+}\right)$; analysis calcd for $\mathrm{C}_{33} \mathrm{H}_{41} \mathrm{ClN}_{4} \mathrm{O}_{2}$ (561.17): C 70.63, H 7.36, N 9.98; found: C 70.44, H 7.56, N 9.63.

\section{3,6-Bis(diethylamino)-9[2-(diethylamino)carbonyl]- xanthylium chloride (8)}

Yield: $472 \mathrm{mg}$ (88\%); m.p. $139-141{ }^{\circ} \mathrm{C} ; R_{F}=0.34\left(\mathrm{SiO}_{2}\right.$, $\mathrm{CHCl}_{3} / \mathrm{MeOH}, 9: 1$ ); IR (ATR): $\nu=3356 \mathrm{w}, 3061 \mathrm{w}, 2971 \mathrm{~m}$, $2932 \mathrm{w}, 2873 \mathrm{w}, 1646 \mathrm{~m}, 1615 \mathrm{~s}, 1586 \mathrm{~s}, 1553 \mathrm{~m}, 1527 \mathrm{~m}$, $1509 \mathrm{~m}, 1481 \mathrm{~m}, 1465 \mathrm{~s}, 1450 \mathrm{~m}, 1430 \mathrm{~s}, 1412 \mathrm{~s}, 1395 \mathrm{~s}$, $1379 \mathrm{~s}, 1335 \mathrm{~s}, 1274 \mathrm{~s}, 1247 \mathrm{~s}, 1196 \mathrm{~m}, 1178 \mathrm{~s}, 1131 \mathrm{~s}, 1076 \mathrm{~s}$, $1009 \mathrm{~s}, 978 \mathrm{~m}, 959 \mathrm{~m}, 921 \mathrm{~m}, 855 \mathrm{~m}, 821 \mathrm{~m}, 789 \mathrm{~m}, 770 \mathrm{~m}$, $761 \mathrm{~m}, \quad 682 \mathrm{~s}, \quad 666 \mathrm{~m}, \quad 626 \mathrm{~m}, \quad 618 \mathrm{~m} \mathrm{~cm}^{-1} ;{ }^{1} \mathrm{H} \quad \mathrm{NMR}$ $\left(400 \mathrm{MHz}, \mathrm{CDCl}_{3}\right): \delta=7.58-7.53(\mathrm{~m}, 2 \mathrm{H}, \quad 5 \mathrm{H}+6 \mathrm{H})$, 7.44-7.40 (m, 1H, 7H), 7.27-7.24 (m, 1H, 8H), 7.15 (d, $J$ $\left.=9.5 \mathrm{~Hz}, 2 \mathrm{H}, 12 \mathrm{H}+12^{\prime} \mathrm{H}\right), 6.86(\mathrm{dd}, J=9.6,2.5 \mathrm{~Hz}, 2 \mathrm{H}$, $\left.13 \mathrm{H}+13^{\prime} \mathrm{H}\right), 6.66\left(\mathrm{~d}, J=2.5 \mathrm{~Hz}, 2 \mathrm{H}, 15 \mathrm{H}+15^{\prime} \mathrm{H}\right), 3.55$ $\left(\mathrm{q}, J=7.2 \mathrm{~Hz}, 8 \mathrm{H}, \quad 17 \mathrm{H}+17^{\prime} \mathrm{H}+17^{\prime \prime} \mathrm{H}+17^{\prime \prime \prime} \mathrm{H}\right), 3.06$ $\left(\mathrm{dq}, J=14.4,7.0 \mathrm{~Hz}, 4 \mathrm{H}, 2 \mathrm{H}+2^{\prime} \mathrm{H}\right), 1.21(\mathrm{t}, J=7.1 \mathrm{~Hz}$, $\left.12 \mathrm{H}, \quad 18 \mathrm{H}+18^{\prime} \mathrm{H}+18^{\prime \prime} \mathrm{H}+18^{\prime \prime \prime} \mathrm{H}\right), 0.99(\mathrm{t}, \quad J=7.0 \mathrm{~Hz}$, $3 \mathrm{H}, 1 \mathrm{H}), 0.52\left(\mathrm{t}, J=7.0 \mathrm{~Hz}, 3 \mathrm{H}, 1{ }^{\prime} \mathrm{H}\right) \mathrm{ppm} ;{ }^{13} \mathrm{C} \mathrm{NMR}$ $\left(101 \mathrm{MHz}, \mathrm{CDCl}_{3}\right): \delta=167.8(\mathrm{C}-3), 157.6(\mathrm{C}-4), 155.9$ $\left(\mathrm{C}-16+\mathrm{C}-16^{\prime}\right), 155.5\left(\mathrm{C}-14+\mathrm{C}-14^{\prime}\right), 136.6$ (C-9), 132.1 (C-7), 130.2 (C-12+C-12'), 130.0 (C-5), 129.9 (C-6), 129.4 (C-8), 126.5 (C-10), 113.9 (C-13+C-13'), $113.6(\mathrm{C}-11+$
C-11'), $96.1\left(\mathrm{C}-15+\mathrm{C}-15^{\prime}\right), 46.1\left(\mathrm{C}-17+\mathrm{C}-17^{\prime}+\mathrm{C}-17^{\prime \prime}\right.$ $\left.+\mathrm{C}-17^{\prime \prime \prime}\right), 43.4(\mathrm{C}-2), 38.3\left(\mathrm{C}-2^{\prime}\right), 14.0(\mathrm{C}-1), 12.6(\mathrm{C}-$ $\left.18+\mathrm{C}-18^{\prime}+\mathrm{C}-18^{\prime \prime}+\mathrm{C}-18^{\prime \prime \prime}\right), 11.6\left(\mathrm{C}-1^{\prime}\right) \mathrm{ppm}$; MS (ESI, $\mathrm{MeOH}): m / z=498.4\left(100 \%,[\mathrm{M}]^{+}\right)$; analysis calcd for $\mathrm{C}_{32} \mathrm{H}_{40} \mathrm{ClN}_{3} \mathrm{O}_{2}$ (534.14): C 71.96, H 7.55, N 7.87; found: C 71.70, H 7.78, N 7.69.

\section{3,6-Bis(diethylamino)-9[2-(1-piperazinyl)carbonyl]- xanthylium chloride (9)}

Yield: (724 mg, 67\%); m.p. $>250{ }^{\circ} \mathrm{C} ; R_{F}=0.14$ (chloroform $/$ methanol, 8:2); IR (ATR) $v=3401 \mathrm{br}, 1589 \mathrm{~m}, 1529 \mathrm{w}$, $1411 \mathrm{~s}, 1328 \mathrm{~s}, 1275 \mathrm{~s}, 1246 \mathrm{~m}, 1180 \mathrm{~s}, 1132 \mathrm{~m}, 1074 \mathrm{~m}, 1011 \mathrm{w}$, 977m, 922m, 820m, 683m; ${ }^{1} \mathrm{H}$ NMR (500 MHz, $\left.\mathrm{CD}_{3} \mathrm{OD}\right): \delta$ $=7.79-7.74(m, 3 \mathrm{H}, 3 \mathrm{H}+4 \mathrm{H}+5 \mathrm{H}), 7.52(\mathrm{~m}, 1 \mathrm{H}, 6 \mathrm{H})$, $7.28-7.25(d, 1 \mathrm{H}, 10 \mathrm{H}), 7.10-7.09(m, 1 \mathrm{H}, 11 \mathrm{H}), 6.98-6.97$ $(d, 1 \mathrm{H}, 13 \mathrm{H}), 3.72-3.59\left(m, 6 \mathrm{H}, 15 \mathrm{H}_{\mathrm{a}}+15 \mathrm{H}_{\mathrm{b}}+17 \mathrm{H}_{\mathrm{a}}+17 \mathrm{H}_{\mathrm{b}}\right.$ $\left.+20 \mathrm{H}_{\mathrm{a}}+20 \mathrm{H}_{\mathrm{b}}\right), 3.08-3.05\left(t, 4 \mathrm{H}, 18 \mathrm{H}_{\mathrm{a}}+18 \mathrm{H}_{\mathrm{b}}+19 \mathrm{H}_{\mathrm{a}}+\right.$ $\left.19 \mathrm{H}_{\mathrm{b}}\right), 1.33-1.30\left(t, 3 \mathrm{H}, 16 \mathrm{H}_{\mathrm{a}}+16 \mathrm{H}_{\mathrm{b}}+16 \mathrm{H}_{\mathrm{c}}\right) \mathrm{ppm} ;{ }^{13} \mathrm{C}$ NMR (126 MHz, $\left.\mathrm{CD}_{3} \mathrm{OD}\right): \delta=169.53(\mathrm{C}-1), 159.2(\mathrm{C}-8)$, 157.3 (C-12), 156.7 (C-14), 135.7 (C-7), 133.0 (C-10), 132.3 (C-2), 131.8 (C-6), 131.5 (C-5), 131.4 (C-4), 128.9 (C-3), 115.4 (C-11), 114.8 (C-9), 97.4 (C-13), 46.9 (C-15), 46.8 (C17+C-20), 44.5 (C-18+C-19), 12.8 (C-16) ppm; MS (ESI, $\mathrm{MeOH}): m / z=256.2\left(24 \%,[\mathrm{M}+\mathrm{H}]^{2+}\right), 511.4(100 \%$, $[\mathrm{M}]^{+}$); analysis calcd for $\mathrm{C}_{32} \mathrm{H}_{39} \mathrm{ClN}_{4} \mathrm{O}_{2}$ (547.14): C 70.25, H 7.18, N 10.24; found: C 70.01, H 7.34, N 10.02.

Acknowledgements We like to thank Dr. D. Ströhl and his team for the NMR spectra and the late Dr. R. Kluge for measuring the MS spectra. Many thanks are also due to V. Simon and M. Schneider for taking the IR spectra. The microanalyses have been determined by S. Ludwig and M. Schneider. The cell lines have been provided by the Department of Oncology (Martin-Luther-University Halle Wittenberg). Open Access funding provided by Projekt DEAL.

\section{Compliance with ethical standards}

Conflict of interest The authors declare that they have no conflict of interest.

Publisher's note Springer Nature remains neutral with regard to jurisdictional claims in published maps and institutional affiliations.

Open Access This article is licensed under a Creative Commons Attribution 4.0 International License, which permits use, sharing, adaptation, distribution and reproduction in any medium or format, as long as you give appropriate credit to the original author(s) and the source, provide a link to the Creative Commons license, and indicate if changes were made. The images or other third party material in this article are included in the article's Creative Commons license, unless indicated otherwise in a credit line to the material. If material is not included in the article's Creative Commons license and your intended use is not permitted by statutory regulation or exceeds the permitted use, you will need to obtain permission directly from the copyright holder. To view a copy of this license, visit http://creativecommons. org/licenses/by/4.0/. 


\section{References}

Beija M, Afonso CAM, Farinha JPS, Charreyre M-T, Martinho JMG (2011) Novel Malachite Green- and Rhodamine B-labeled cationic chain transfer agents for RAFT polymerization. Polymer 52:5933-5946

Bui DT, Nicolas J, Maksimenko A, Desmaele D, Couvreur P (2014) Multifunctional squalene-based prodrug nanoparticles for targeted cancer therapy. Chem Commun 50:5336-5338

Costantini P, Jacotot E, Decaudin D, Kroemer G (2000) Mitochondrion as a novel target of anticancer chemotherapy. J Natl Cancer Inst 92:1042-1053

Csuk R, Deigner H-P (2019) The potential of click reactions for the synthesis of bioactive triterpenes. Bioorg Med Chem Lett 29:949-958

Dauner M, Batroff E, Bachmann V, Hauck CR, Wittmann V (2016) Synthetic Glycosphingolipids for Live-Cell Labeling. Bioconjugate Chem 27:1624-1637

Del Secco B, Malachin G, Milli L, Zanna N, Papini E, Cornia A, Tavano R, Tomasini C (2017) Form matters: stable helical foldamers preferentially target human monocytes and granulocytes. ChemMedChem 12:337-345

Fulda S (2010) Exploiting mitochondrial apoptosis for the treatment of cancer. Mitochondrion 10:598-603

Galluzzi L, Larochette N, Zamzami N, Kroemer G (2006) Mitochondria as therapeutic targets for cancer chemotherapy. Oncogene 25:4812-4830

Gogvadze V, Orrenius S, Zhivotovsky B (2009a) Mitochondria as targets for cancer chemotherapy. Semin Cancer Biol 19:57-66

Gogvadze V, Orrenius S, Zhivotovsky B (2009b) Mitochondria as targets for chemotherapy. Apoptosis 14:624-640

Guo R, Yin J, Ma Y, Wang Q, Lin W (2018) A novel mitochondriatargeted rhodamine analogue for the detection of viscosity changes in living cells, zebra fish and living mice. J Mater Chem B 6:2894-2900

Johnson LV, Walsh ML, Chen LB (1980) Localization of mitochondria in living cells with rhodamine-123. Proc Natl Acad Sci-Biol 77:990-994

Kahnt M, Wiemann J, Fischer L, Sommerwerk S, Csuk R (2018) Transformation of asiatic acid into a mitocanic, bimodal-acting rhodamine B conjugate of nanomolar cytotoxicity. Eur J Med Chem 159:143-148

Mai MS, Allison WS (1983) Inhibition of an oligomycin-sensitive ATPase by cationic dyes, some of which are atypical uncouplers of intact mitochondria. Arch Biochem Biophys 221:467-476

May F, Peter M, Huetten A, Prodi L, Mattay J (2012) Synthesis and characterization of photoswitchable fluorescent $\mathrm{SiO} 2$ nanoparticles. Chem Eur J 18:814-821

Melikyan GB, Dieriy BN, Ok DC, Cohen FS (1996) Voltagedependent translocation of R18 and Dil across lipid bilayers leads to fluorescence changes. Biophys J 71:2680-2691

Mottram LF, Forbes S, Ackley BD, Peterson BR (2012) Hydrophobic analogues of rhodamine B and rhodamine 101: potent fluorescent probes of mitochondria in living $C$. elegans. Beilstein $\mathrm{J}$ Org Chem 8:2156-2165. No. 2243

Neuzil J, Dong L-F, Rohlena J, Truksa J, Ralph SJ (2013) Classification of mitocans, anti-cancer drugs acting on mitochondria. Mitochondrion 13:199-208

Oprea TI (2002) Virtual screening in lead discovery: a viewpoint. Molecules 7:51-62

Preston AN, Farr JD, O’Neill BK, Thompson KK, Tsirka SE, Laughlin ST (2018) Visualizing the brain's astrocytes with diverse chemical scaffolds. ACS Chem Biol 13:1493-1498

Rashid F, Horobin RW (1990) Interaction of molecular probes with living cells and tissues. Part 2. A structure-activity analysis of mitochondrial staining by cationic probes, and a discussion of the synergistic nature of image-based and biochemical approaches. Histochemistry 94:303-308

Rokitskaya TI, Klishin SS, Severina II, Skulachev VP, Antonenko YN (2008) Kinetic analysis of permeation of mitochondria-targeted antioxidants across bilayer lipid membranes. J Membr Biol 224:9-19

Rokitskaya TI, Korshunova GA, Antonenko YN (2018) Effect of alkyl chain length on translocation of rhodamine B n-alkyl esters across lipid membranes. Biophys J 115:514-521

Shakurova ER, Pozdnyakova DA, Tretyakova EV, Parfenova LV (2020) One-pot synthesis of betulin triterpenoid quaternized pyridine derivatives and their antimicrobial activity. Lett Drug Des Discov 17:79-84

Sodano F, Gazzano E, Fraix A, Rolando B, Lazzarato L, Russo M, Blangetti M, Riganti C, Fruttero R, Gasco A, Sortino S (2018) A molecular hybrid for mitochondria-targeted NO photodelivery. ChemMedChem 13:87-96

Sommerwerk S, Heller L, Kerzig C, Kramell AE, Csuk R (2017) Rhodamine $\mathrm{B}$ conjugates of triterpenoic acids are cytotoxic mitocans even at nanomolar concentrations. Eur J Med Chem 127:1-9

Song S, Gao K, Niu R, Yi W, Zhang J, Gao C, Yang B, Liao X (2019) Binding behavior, water solubility and in vitro cytotoxicity of inclusion complexes between ursolic acid and amino-appended $\beta$-cyclodextrins. J Mol Liq 296:111993

Talib AJ, Fisher A, Voronine DV, Sinyukov AM, Bustamante Lopez SC, Ambardar S, Meissner KE, Scully MO, Sokolov AV (2019) Fluorescence imaging of stained red blood cells with simultaneous resonance Raman photostability analysis. Analyst 144:4362-4370

Tansil NC, Li Y, Koh LD, Peng TC, Win KY, Liu XY, Han M-Y (2011) The use of molecular fluorescent markers to monitor absorption and distribution of xenobiotics in a silkworm model. Biomaterials 32:9576-9583

Walters WP, Ajay, Murcko MA (1999) Recognizing molecules with drug-like properties. Curr Opin Chem Biol 3:384-387

Wang D, Wang Z, Li Y, Song Y, Song Y, Zhang M, Yu H (2018) A single rhodamine spirolactam probe for localization and $\mathrm{pH}$ monitoring of mitochondrion/lysosome in living cells. N. J Chem 42:11102-11108

Wieker HJ, Kuschmitz D, Hess B (1987) Inhibition of yeast mitochondrial F1-ATPase, F0F1-ATPase and submitochondrial particles by rhodamines and ethidium bromide. Biochim Biophys Acta Bioenerg 892:108-117

Wiemann J, Fischer L, Kessler J, Ströhl D, Csuk R (2018) Ugi multicomponent-reaction: Syntheses of cytotoxic dehydroabietylamine derivatives. Bioorg Chem 81:567-576

Wolfram RK, Fischer L, Kluge R, Ströhl D, Al-Harrasi A, Csuk R (2018a) Homopiperazine-rhodamine B adducts of triterpenoic acids are strong mitocans. Eur J Med Chem 155:869-879

Wolfram RK, Heller L, Csuk R (2018b) Targeting mitochondria: Esters of rhodamine $\mathrm{B}$ with triterpenoids are mitocanic triggers of apoptosis. Eur J Med Chem 152:21-30

Xie C, Chang J, Hao X-D, Yu J-M, Liu H-R, Sun X (2013) Mitochondrial-targeted prodrug cancer therapy using a rhodamine B labeled fluorinated docetaxel. Eur J Pharm Biopharm 85:541-549

Yu B, Dong C-Y, So PTC, Blankschtein D, Langer R (2001) In vitro visualization and quantification of oleic acid induced changes in transdermal transport using two-photon fluorescence microscopy. J Investig Dermatol 117:16-25

Zhang M, Shen C, Jia T, Qiu J, Zhu H, Gao Y (2020) One-step synthesis of rhodamine-based $\mathrm{Fe} 3+$ fluorescent probes via Mannich reaction and its application in living cell imaging. Spectrochim Acta Part A 231:118105 